\title{
Development of Gait Recognition in NI LabVIEW
}

\author{
Dániel SALÁNKI \\ Department of Mechatronics \\ University of Debrecen \\ Debrecen, Hungary \\ danisalanki@yahoo.com
}

\author{
Kornél SARVAJCZ \\ Department of Mechatronics \\ University of Debrecen \\ Debrecen, Hungary \\ sarvajcz@eng.unideb.hu
}

\begin{abstract}
Nowadays, one of the most significantly improving area in security is the world of biometric identifiers. Within the biometric identifiers, the research group is working with the gait recognition speciality. The research group realized a complex gait recognition system in NI LabVIEW, that can detect more reference points simultaneously with a universal camera and is capable of suiting predetermined curves on the detected points. Moreover, the program can compare the functions suited on the reference curve and the actual curve and evaluate if the two gait images are the same or not. In the program there is a saving and a reloading function which contributes to the production of the reference gait image. The foot analysis program before the gait recognition is designed to improve accuracy. The self-developed gait recognition system was tested on more persons and the False Acceptance Rate (FAR) was zero.
\end{abstract}

Keywords - biometric; gait; recognition; camera; analysis.

\section{INTRODUCTION}

In today's world, I find it important to protect our values with the mechanical and electronic tools at our disposal. As a mechatronics engineer student, I sought to find a theme that plays a decisive role in everyday life and is closely related to mechatronics. Numerous biometric identification methods exist today (e.g. face, voice, palm, iris, retina, fingerprint authentication and DNA analysis), but gait recognition is less common.

This is how we came up with the idea of wanting to implement a camera-based gait recognition program using the Vision module of NI LabVIEW software, which is capable of real-time image processing to recognize that the gait image of a person passing by in front of the system is the same as that of the previously stored person's gait image. If this condition is met, the system gives him / her permission. The gait recognition is preceded by a foot examination that examines the leg/foot ratio of the person. If the test is passed, the actual gait recognition begins.

\section{GAIT RECOGNITION - REFERENCES}

People can often feel that a familiar person is already recognized far from their walk. This common experience is the idea behind the fact that district recognition systems can be used in security technology. You can easily make an image of a person's walk, even in public places, without even getting observed by that person. Walking is also influenced by several factors: footwear, soil, fatigue, current state of mind or any injury [1].

Walking can be defined as a series of cyclic and coordinated movements that result in human displacement [1]. Compared to physiological biometric identification (fingerprint authentication, iris and face identification, earlobe geometry identification), the gait recognition has the advantage of identifying persons with low resolution or hidden camera shots [2]. Physiological biometric methods cannot reliably detect non-cooperating individuals, especially not at distances, under the changing environmental conditions of the real world [3]. However, the accuracy of walking recognition can be greatly influenced by several factors, such as the viewing angle that changes the visual attributes [4].

\section{FOOT ANALYSIS PROGRAM}

The essence of foot analysis is that we use a program developed in LabVIEW to determine the ration of the leg's and foot's lengths. We first measured the ratio of one of our legs, which ranged between 2.42 and 2.43, so we selected these two numbers as limit values. So, if the ratio of the leg and foot measured in pixels is between these two numbers, the program will give a green signal.

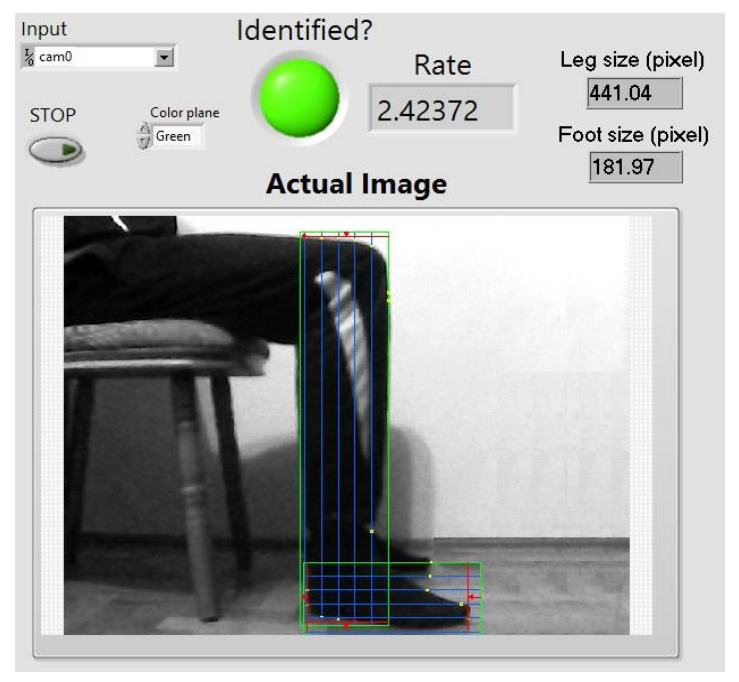


Fig. 1. Foot analysis program during operation

Fig. 1 shows the program after the run. The proportional value (2.42372) corresponded to the set limits, so the green light flashes. During the experiment, the person was sitting in pants and socks, but similar values were achieved in shorts, without socks, so it can be stated that the clothes have minimal influence on the results and therefore are negligible in terms of analysis.

The results of a series of 20 measurements are shown in Fig. 2. Without footwear, the program was working with $90 \%$ accuracy, and in the case of two negative results only $0.5 \%$ was missing for compliance. Wearing footwear (slippers), the program never recognized the person. The simple explanation is that the footwear has larger dimensions than the foot. So, the measurement is necessary to be done without footwear to achieve the correct result. With another person, the leg/foot ratio was around 2.5, so the green light didn't flash.

\begin{tabular}{|c|c|c|c|c|c}
\hline Measurement nr. Leg/Foot ratio & Passed? & Measurement $\mathrm{nr}$. Leg/Foot ratio & Passed? \\
\hline 1 & 2.42384 & yes & 14 & 2.41906 & no \\
2 & 2.42735 & yes & 15 & 2.42288 & yes \\
3 & 2.42863 & yes & 16 & 2.42511 & yes \\
4 & 2.42407 & yes & 17 & 2.42091 & yes \\
5 & 2.42514 & yes & 18 & 2.4181 & no \\
6 & 2.42643 & yes & 19 & 2.42372 & yes \\
7 & 2.42227 & yes & 20 & 2.42337 & yes \\
8 & 2.42124 & yes & 21 & 2.26314 & no \\
9 & 2.42441 & yes & 22 & 2.2617 & no \\
10 & 2.42424 & yes & 23 & 2.26053 & no \\
11 & 2.42138 & yes & 24 & 2.46508 & no \\
12 & 2.42316 & yes & 25 & 2.46502 & no \\
13 & 2.42396 & yes & & & \\
\hline
\end{tabular}

Fig. 2. 20 measurements without footwear (grey fields), 5 measurements in footwear (blue fields)

\section{The Presentation of THE Gait ReCOGNition Software}

We attach a point to the knee and one to the leg of the person, in the tibia region. The background of the points is white, the point itself is black. It is important to have a light or white background to avoid false point detection, and to have your foot closer to the camera where the reference points are located. Measurements were made with artificial light, with normal room lighting, with a darkened shade, creating approximately the same lighting conditions for each measurement.

Fig. 3 shows the front panel after running. On the right side of the figure, the coordinates of the detected points are stored in blocks. On the second and third tabs, the gait image is processed. On the second tab, the actual gait image is processed, while on the third tab the saved and reloaded gait image is processed. The program suits a function on the curves drawn by the two reference points (see Fig. 4). You can choose between four function models (Linear, Polynomial, Exponential, Power - see Fig. 4) and you can choose the order of the function too. On Fig. 4 the blue points represent the detected points and the red curves are the functions suited on them. According to the tests, the most accurate fit is the fourth-degree (quartic) polynomial function. However, for quarter-degree polynomials, the third and fourth coefficients take very small values (up to $10^{-10}$ ), and the second-order (quadratic) function also follows well the curve drawn by the points, so the use of the fourth-degree polynomials becomes unnecessary.

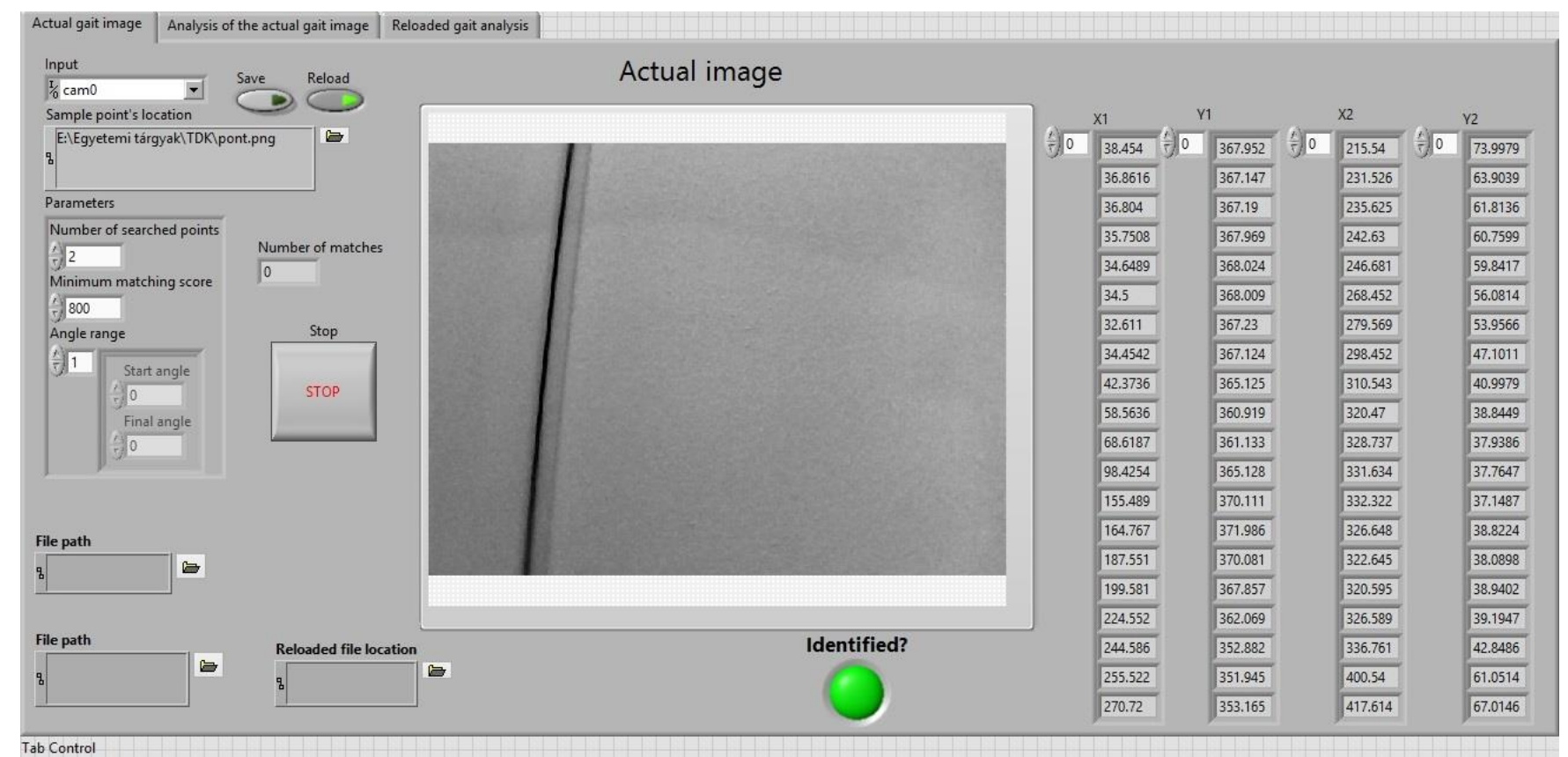

Fig. 3. The front panel of the gait recognition software 


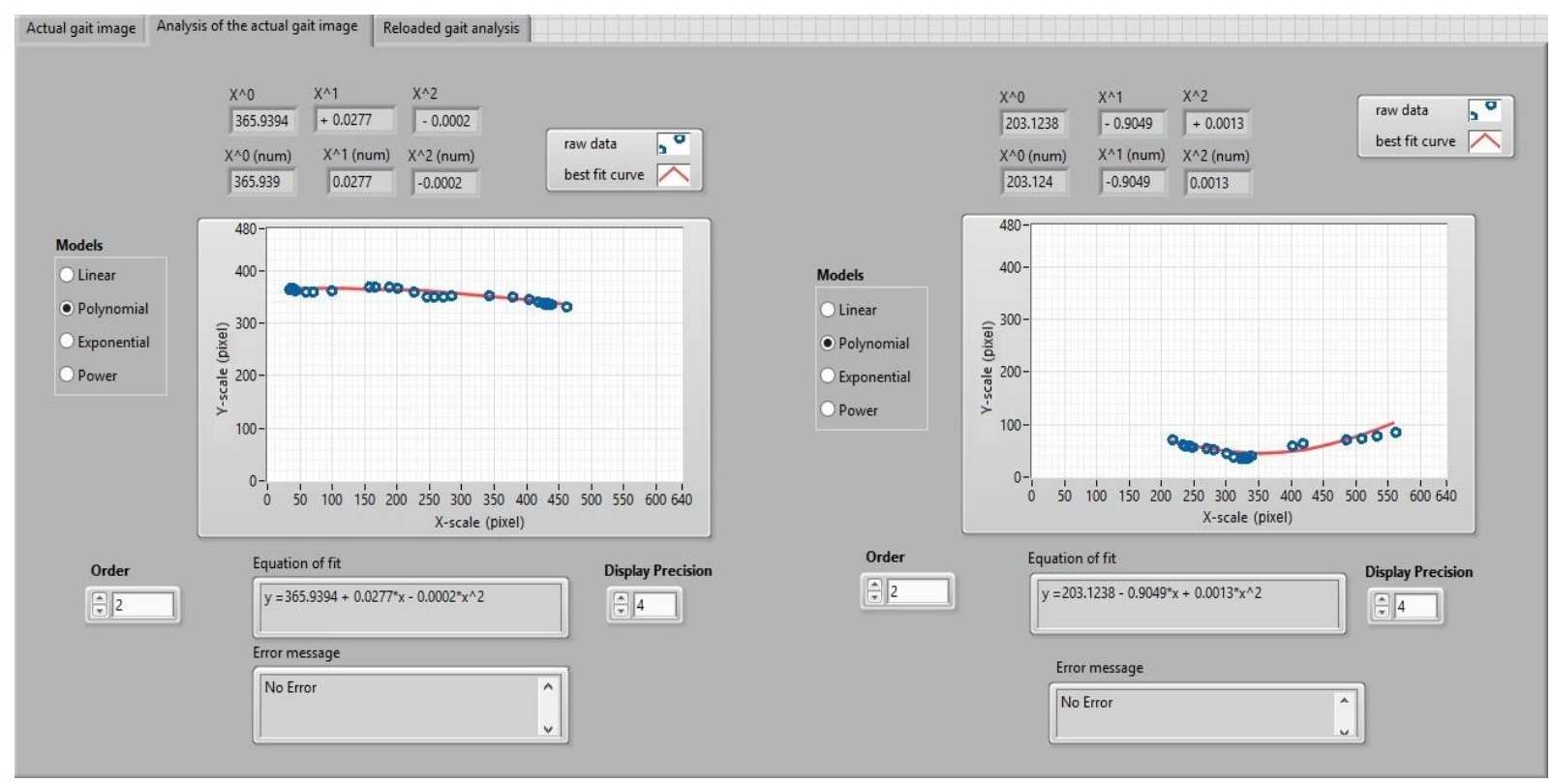

Fig. 4. Second tab - The detected points and the functions suited on curves

How can two gait patterns be compared? Before testing, the coefficients of a previously saved gait pattern are reloaded, and we measure the current gait pattern. For zero-degree coefficients, if the deviation is within $5 \%$ (first point) or $25 \%$ (second point), the program fragment provides one logic 1 . For the first-degree and second-degree coefficients, we have set boundaries because of the high percentage deviations, shown in Table I. If within that narrow bounds falls the given coefficient, it also gives a logical 1 to the program. If all six of the logic 1s are given, the green light will flash, so the system recognized the person.

TABLE I.

\begin{tabular}{|c|c|c|c|c|c|c|c|}
\hline \multicolumn{4}{|c|}{ First point } & \multicolumn{3}{c|}{ Second point } \\
\hline \multicolumn{2}{|c|}{$X^{\wedge} 2$} & \multicolumn{2}{|c|}{$X^{\wedge} 1$} & \multicolumn{2}{c|}{$X^{\wedge} 2$} & \multicolumn{2}{c|}{$X^{\wedge} 1$} \\
\hline Max. & Min. & Max. & Min. & Max. & Min. & Max. & Min. \\
\hline 0.0002 & -0.0003 & 0.05 & -0.14 & 0.002 & 0.001 & -0.5 & -1.2 \\
\hline
\end{tabular}

\begin{tabular}{|c|c|c|c|c|c|c|c|c|c|c|c|}
\hline$x^{\wedge} 0$ & $x^{\wedge} 1$ & $x^{\wedge} 2$ & $x^{\wedge} 0$ & $x^{\wedge} 1$ & $x^{\wedge} 2$ & $x^{\wedge} 0$ & $x^{\wedge} 1$ & $x^{\wedge} 2$ & $x^{\wedge} 0$ & $x^{\wedge 1}$ & $x^{\wedge} 2$ \\
\hline 373.6298 & -0.2329 & 0.0002 & 325.4167 & -1.3881 & 0.0025 & 353.6152 & -0.1192 & $8.26 \mathrm{E}-05$ & 139.1351 & -0.5073 & 0.0009 \\
\hline 373.3425 & -0.1974 & 0.0001 & 196.1278 & -0.6277 & 0.0011 & 353.7429 & -0.0867 & $-3.49 E-05$ & 170.9622 & -0.7358 & 0.0012 \\
\hline 379.34 & -0.2399 & 0.0002 & 249.1721 & -0.9672 & 0.0016 & 355.5487 & -0.0672 & $-9.52 E-05$ & 196.8606 & -0.858 & 0.0013 \\
\hline 372.077 & -0.1939 & $8.97 E-05$ & 219.6966 & -0.8317 & 0.0015 & 361.3664 & -0.1049 & $-4.14 E-05$ & 174.3736 & -0.6982 & 0.0011 \\
\hline 378.0727 & -0.264 & 0.0002 & 236.9901 & -0.9307 & 0.0016 & 357.2629 & -0.1118 & $4.16 \mathrm{E}-05$ & 162.301 & -0.6797 & 0.0012 \\
\hline 380.2123 & -0.2756 & 0.0003 & 147.7936 & -0.2505 & 0.0004 & 362.4711 & -0.1184 & $2.62 E=05$ & 185.2648 & -0.7772 & 0.0013 \\
\hline 376.068 & -0.2785 & 0.0003 & 222.5547 & -0.839 & 0.0015 & 357.1883 & -0.1124 & $6.12 \mathrm{E}-05$ & 170.0862 & -0.7681 & 0.0014 \\
\hline 370.8115 & -0.2147 & 0.0002 & 152.7052 & -0.2911 & & 344.6318 & 0.0053 & -0.0002 & 181.6022 & -0.7892 & .0013 \\
\hline 384.1069 & -0.2987 & 0.0003 & 172.5713 & -0.3834 & .0006 & 357.0924 & -0.0731 & $.50 E-05$ & 196.4917 & -0.892 & .0015 \\
\hline 369.9979 & -0.2017 & 0.0001 & 214.1039 & -0.7529 & 0.0013 & 379.9573 & -0.2489 & 0.0003 & 234.2505 & -1.1582 & 0.0019 \\
\hline
\end{tabular}

Fig. 5. The coefficients of the suited functions on the detected points

On Fig. 5, the green columns refer to the first point, while the blue columns refer to the second point. The six columns on the left are the values of one person, while the right-hand six columns refer to another person with similar height. Based on the results, in 8 of 10 cases the tested person was detected by the system correctly, while the False Acceptance Rate was zero, so the gait recognition system never accepted an access attempt by an unauthorized user.

\section{CONCLUSION}

In conclusion, the accuracy of the district recognition system is adequate and, in combination with the previous foot analysis program, its accuracy can be improved further by implementing a two-stage biometric system. Plans in the future include more cameras to get a better image of a person's gait. The cameras could be positioned at a certain angle and coordinated with the images formed by them to obtain a camera system that can be examined with spatial geometric equations. In addition, we would like to introduce additional reference points, thereby reducing the defect potential during detection.

\section{Acknowledgment}

The publication / presentation / poster was supported by the EFOP-3.6.1-16-2016-00022 project. The project was funded by the European Union, co-financed by the European Social Fund.

\section{References}

[1] Jeffrey E. Boyd, James J. Little: Biometric Gait Recognition Springer - Verlag Berlin Heidelberg 2005, 19-42.

[2] Mark S. Nixon, T. N. Tan, R. Chellappa: Human Identification Based on Gait, Springer Science + Business Media, Inc., New York, USA, 2006.

[3] Ju Han, Bir Bhanu: Individual Recognition Using Gate Energy Image, IEEE transactions on pattern analysis and machine intelligence 28.2: 316-322, 2006.

[4] Wei Zeng, Cong Wang: Neurocomputing - View-invariant gait recognition via deterministic learning, 175: 324-335, 2016. 